\title{
Prognostic Significance of Pretreatment Variables in Patients with Invasive Cervical Cancer
}

\author{
E.R. TE VELDE, ${ }^{*}$ J.D.F. HABBEMA, $\dagger$ G.J. GELPKE $\dagger$ and R.E. BALLIEUX+ \\ Departments of Gynecology* and Clinical Immunology, ${ }_{+}^{+}$University Hospital, Utrecht and the Department of Public Health and \\ Social Medicine, $\dagger$ Erasmus University, Rotterdam, The Netherlands
}

\begin{abstract}
In 119 consecutive patients with invasive cervical cancer 92 variables of potential significance in predicting recurrence were determined before treatment. In 49 patients recurrent disease was diagnosed during follow-up after therapy. The investigated variables include: clinical data, routinely performed hematological and biochemical tests, histopathological data, oncofetal antigens, placental and pregnancy associated substances, serum levels of acute phase reactants, complement components, immunoglobulins and circulating immune complexes, and assays measuring the general immune competence of the patient. Thirty-three of the variables appeared to give statistically significant differences between values of future recurrence and non-recurrence patients. Further selection was carried out by stepwise forward discriminant analysis. This selection resulted in a prognosis rule using a subset of 7 variables. Carcino-embryonic antigen and the complement component C3PA appeared to be the most powerful variables. Although the stage of disease was the third most important single variable, it did not add prognostic information, once CEA and C3PA were used. By applying the prognosis rule a probabilistic prediction is obtained for each patient. In about $60 \%$ of the future recurrences and $40 \%$ of the future non-recurrence patients, these predictions reached a high level of accuracy. It was argued that the prediction of nonrecurrence does not warrant to change the treatment protocol at present. Whether the prediction of recurrence is clinically useful remains to be clarified.
\end{abstract}

\section{INTRODUCTION}

FIVE-YEAR survival rates of patients treated for macro-invasive cervical cancer have hardly improved during the last two decades [1]. The treatment protocol has been primarily determined by the extent of disease as estimated by clinical staging. Within a given stage each patient is treated identically, although in addition to the stage of disease, several prognostic factors have been reported [2-10]. These findings have not resulted in a further differentiation of the treatment protocol adjusted to the individual prognosis of the patient. Apparently, reliable prognostic factors which are valid for the individual patient are lacking so far. The purpose of this study is to investigate the possibility of making accurate pretreatment predictions as to the course of the individual patient, based on a systematic selection of combinations of

Accepted 8 May 1987.

Supported by a grant from the Netherlands Cancer Society (Koningin Wilhelmina Fonds).

Address for reprints: E.R. te Velde, Department of Gynecology, University Hospital, Catharijnesingel 101, 3511 GV Utrecht, The Netherlands. prognostic variables, and of designing a clinical prediction aid.

\section{MATERIALS AND METHODS}

A. Patients

One hundred and nineteen consecutive patients with macro-invasive cancer of the uterine cervix were studied in the University Hospital of Utrecht. All tumors were histologically proven squamous carcinomas varying from non-differentiated to welldifferentiated types. The methods of clinical staging, the modes of therapy, the follow-up schedule and the establishment and site of recurrence have been described previously $[11,12]$. The number of recurrences and non-recurrences for the various tumor stages are shown in Table 1. The median time between therapy and the clinical diagnosis of recurrence was 48 weeks (range 18-218). The median follow-up time of the non-recurrences at the time of evaluation was 221 weeks (range 130-312).

B. Variables of potential prognostic significance

Seventy four variables were measured before any treatment was given. 
Table 1. Recurrences and age of the patients according to the clinical tumor stage

\begin{tabular}{llllll}
\hline Stages (FIGO) & I & IIA & IIB & III & All stages \\
\hline Number of patients & 38 & 15 & 31 & 35 & 119 \\
Non-recurrences & 31 & 8 & 15 & 16 & 70 \\
Recurrences $(\%)$ & $7(18 \%)$ & $7(47 \%)$ & $16(52 \%)$ & $19(54 \%)$ & $49(41 \%)$ \\
Median age in years & 49 & 55 & 59 & 60 & 56 \\
(range) & $(25-75)$ & $(38-81)$ & $(35-79)$ & $(33-85)$ & $(25-85)$ \\
\hline
\end{tabular}

Clinical data. Age, menopause, tumor stage, smoking habits, second malignancy, cervical carcinoma in first degree family, non-malignant disease, the number of pregnancies, the time of the last pregnancy.

Routinely performed hematological and biochemical tests. The blood group, erythrocyte sedimentation rate, hemoglobin, hematocrit, \% reticulocytes, numbers of: erythrocytes, thrombocytes and leukocytes; differentiation of leukocytes: \% of neutrophils, \% bandforms, \% lymphocytes, $\%$ eosinophils, $\%$ monocytes; kidney function: serum levels of creatinine and ureum; liver function: serum levels of alkaline phosphatase, lactate dehydrogenase, serum glutamate oxaloacetate transaminase, serum glutamate pyruvatc transaminase and gamma glutamyl transferase; total serum protein level, electrophoretic determination of serum protein fractions: albumin, alpha-1-globulin, alpha-2-globulin, beta-globulin, gamma-globulin.

Histopathological data. Cell differentiation (non-, poorly, moderately and well-differentiated), histological classification according to Wentz and Reagan [13] (keratinizing carcinomas, large cell non-keratinizing carcinomas and small cell carcinomas), degree of subepithelial mononuclear cell infiltration, the mode of tumor infiltration, the presence of tumor invasion in lymphovascular spaces.

Oncofetal antigens. Serum levels of carcinoembryonic antigen, alpha-fetoprotein.

Placental and pregnancy-associated substances. Serum levels of human chorionic gonadotrophin, carcinoplacental alkalinc phosphatase, alpha-2-pregnancyassociated macroglobulin and the soluble placenta proteins PP 7 and PP 8.

Some acute phase reactants and complement components. Serum levels of haptoglobin, alpha-1-acidglycoprotein, C-reactive protein, alpha-1-antitrypsin, C4 and C3-proactivator, C1-esterase inhibitor and $\mathrm{C} 9$.
Immunoglobulin serum levels. IgM, IgG, IgA and IgD.

Levels of circulating immune complexes in serum as detected by $\mathrm{C} I Q$ and conglutinin binding assays or determined by inhibition of rheumatoid factor and an agglutinating factor in mouse serum.

Lymphocyte proliferative assays using phytohemagglutinin at optimal and suboptimal concentrations, pokeweed mitogen, a cocktail of various antigens and allogeneic lymphocytes as stimulator cells.

Determination of NK-K cell lytic capacity.

Determination of lymphocyte subpopulations ( $T$ and $B$ cells).

Determination of suppressive activity of patients' serum on PHA-induced proliferation of donor lymphocytes.

In addition 18 variables were derived by combination of various of the above mentioned, adding up to 92 variables. They only include combinations which are generally used in clinical medicine and immunology. For example, the number of lymphocytes, one of the combined variables, was obtained by multiplying the \% lymphocytes and the number of leucocytes. The laboratory methods used and the justification for their performance are described elsewhere [12]. All data were entered into a database for statistical evaluation.

\section{Statistical methods}

The endpoint of interest in this population of cervical cancer patients is whether or not patients will experience recurrence of disease. Pretreatment data of the patients are related to these two endpoints in order to establish whether a difference in pretreatment values is prognostically meaningful. Since the last recurrence was established at 218 weeks after therapy and the follow-up for nonrecurrence patients varied from 130 wecks to 312 weeks, some additional recurrences may occur. By using life-table analysis we estimate that in the present non-recurrence group of 70 , about two patients are to be expected to experience recurrent disease within the maximum follow-up time of 6 years. This small number is consistent with the knowledge that a recurrence in cervical cancer is relatively rare after $2 \frac{1}{2}$ years of follow-up and it only 
slightly influences the results of our analysis. For the establishment of an individual prognosis for each patient, we used discriminant analysis. Proportional hazard analysis or 'cox regression' has also been considered, but its assumption of a constant relative risk of each prognostic factor over time, was not satisfied in our problem.

1. Screening of the pretreatment data. A first selection was performed, by comparing the pretreatment values of the 49 recurrence with those of the 70 non-recurrence patients, using simple parametric (Student's $t$-test) and non-parametric (Wilcoxon's test) statistical significance tests. The categorized variables (most clinical and all histological data) were assessed by chi-square tests. When statistically significant differences $(P<0.05$, one-sided) were obtained with at least one of the statistical tests, the variable was considered to be of potential prognostic value and was used for further cvaluation.

2. Prognosis rule. A second selection was carried out by stepwise discriminant analysis.

Such an analysis results in a prognosis rule which gives a probabilistic prediction for each patient. The prediction depends on the assumptions about the statistical model of the data. The 'independence model', as discussed by Lusted [14], was used. The assumptions underlying this model are only partly true, since there is interaction between some of the variables studied. However, in many situations more sophisticated statistical models have not resulted in more accurate predictions [15]. Since a computer program for a variable selection with the independence model is only available for categorical data, continuous variables were recoded into five categories. The boundaries between the categories were chosen at the 1/9, 3/9, 6/9 and $8 / 9$ fractiles of the sample distribution of each variable.

Which variables are to be used for prediction? To use simply all available data is probably the worst possible solution, especially when the number of variables is large and the number of patients is limited. The aim is to select a small subset of variables containing (nearly) all of the prognostic information, as measured by the quality of the corresponding probabilistic prognosis rule. The INDEP-SELECT computer program has been used for this selection $[17,18]$. The procedure runs as follows: in step 1 , the one variable is selected that in itself yields the highest quality of prediction. In step 2, from all the remaining variables a second one is selected that contains-in combination with the variable from step 1-the highest quality of prediction. In the third and subsequent steps, variables are added to the set, until no further improvement in quality takes place. The prognosis rule is based on these selected variables. Prognostic probabilities for both outcome categories can be calculated from this rule for all patients. For example, the probability of recurrence in a given patient is predicted to be $80 \%$. Consequently the probability of non-recurrence is $20 \%$, because the probabilities have to add to $100 \%$.

\section{Quality measures of the prognosis rule.}

3.1. Error rate. The quality of a prognosis rule is usually measured by the error rate, i.c. the proportion of patients which were not assigned to the actual outcome category. However, the error rate is a rough reflection of the probabilities: a probability of $60 \%$ for the actual outcome category is judged to be equally good as for example $90 \%$.

3.2. Modified logarithmic score. Other measures take the precise value of the probabilities into account [19], one of them being the so-called modified logarithmic score [20]. It assigns a different wcight to a probability of $90 \%$ as compared to a probability of $60 \%$.

3.3. Sharp predictions. Predictions approaching the $50-50$ level will be less accurate than the socalled 'sharp predictions' in which the probability allocated to one category exceeds a high (arbitrarily chosen) threshold level. Predictions below this level are then no longer taken into consideration, remaining in the shadow of doubt [21].

3.4. Cross-validation. The quality of the prognosis rule is overestimated because prediction probabilities for a patient are partly based on her own information as a reference patient. To avoid this bias, the patients were randomly split into 10 groups. The whole procedure for obtaining a prognosis rule was executed on the patient data from nine out of the 10 groups. The resulting prognosis rule was applied to each patient of the 10th group, now being a really independent test-group. This method of crossvalidation (designated as 'split-ten method') was repeated 10 times so that each patient was a test-patient once.

\section{RESULTS}

\section{A. Single variables}

Thirty-three of the 92 variables had a favorable screening result. In Table 2 the top 10 variables are ordered by the criterion score uscd for sclcction (modified $\log$ ), but the error rate is also given. The serum level of CEA, not the stage of disease, appears to be the best single factor discriminating recurrences from non-recurrences. The values for the modified $\log$ in Table 2 can be contrasted with a value of 0.63 that can be calculated when a $41 \%$ 
Table 2. The 10 single variables which yield the best prediction of (non-) recurrence

\begin{tabular}{lccc}
\hline Variables & $\begin{array}{c}\text { Rank } \\
\text { No. }\end{array}$ & $\begin{array}{c}\text { Modified } \\
\text { log-score }\end{array}$ & $\begin{array}{c}\text { Error } \\
\text { rate }\end{array}$ \\
\hline Carcino-embryonic antigen (CEA) & 1 & 0.558 & 0.33 \\
C $_{3}$-proactivator $\left(\mathrm{C}_{3} \mathrm{PA}\right)$ & 2 & 0.589 & 0.42 \\
Stage of disease & 3 & 0.607 & 0.35 \\
\% Leucocytes with bandforms & 4 & 0.622 & 0.35 \\
Alpha-1-globulin (proportion) & 5 & 0.625 & 0.37 \\
Cystoscopy & 6 & 0.631 & 0.47 \\
Age & 7 & 0.631 & 0.39 \\
Lactate dehydrogenase & 8 & 0.632 & 0.51 \\
Number of leucocytes with bandforms & 9 & 0.633 & 0.37 \\
Alkaline phospatase & 10 & 0.633 & 0.36 \\
\hline
\end{tabular}

Table 3. Prognostic variables selected by stepwise forward discriminant analysis

\begin{tabular}{llcc}
\hline $\begin{array}{l}\text { Selection } \\
\text { step }\end{array}$ & $\begin{array}{l}\text { Variable } \\
\text { added }\end{array}$ & $\begin{array}{c}\text { Criterion } \\
\text { score }\end{array}$ & $\begin{array}{c}\text { Error } \\
\text { rate }\end{array}$ \\
\hline 0 & No variable selected yet & 0.63 & \\
1 & CEA & 0.56 & 0.33 \\
2 & $\mathrm{C}_{3}$ PA & 0.52 & 0.31 \\
3 & \% Bandforms & 0.50 & 0.29 \\
4 & Age of the patient & 0.48 & 0.26 \\
5 & Lymphovascular invasion & 0.46 & 0.23 \\
6 & Reticulocytes & 0.45 & 0.24 \\
7 & Number of eosinophils & 0.44 & 0.25 \\
\hline
\end{tabular}

and $59 \%$ prediction for recurrence respectively for non-recurrence would be assumed according to the proportions in our study. Only the top few variables yield a considerable improvement in prediction quality.

\section{B. Stepwise forward selection}

Table 3 presents the results of the stepwise forward selection process, when using the 33 variables. The criterion score decreases with every step, indicating predictive improvement of the combination of variables by adding the newly selected one. The selection process stops when no variable can add any further improvement in prognostic quality. This appears to happen after selection of the 7 th variable. Note that there is only a slight improvement of the criterion score after the selection of the 4th variable.

\section{Sharp predictions}

The percentages of false predictions when a patient is always classifed ( $50 \%$ level), the percentages of sharp predictions at the arbitrarily taken $80 \%$ and $90 \%$ thresholds, and the percentages of sharp predictions found to be false at these levels are presented in Table 4 . The proportion of patients allocated to the wrong category at the $50 \%$ level hardly decreases by the selection of more variables, but the percentage of sharp predictions increases considerably. The error rate among the sharp predictions varies from $0-10 \%$ and $0-4 \%$ at the $80 \%$ and $90 \%$ level respectively, without a clear trend when the number of the selected variables is increased.

\section{Correctly and falsely predicted recurrences and non- recurrences}

Table 4 presents the total proportion of false predictions at two threshold levels. This proportion is a mixture of two kinds of misclassifications: a true non-recurrence patient is predicted as a recurrence and a true recurrence patient as a nonrecurrence. In Table 5 this distinction is used for calculating the predictive values of recurrence and non-recurrence at various threshold levels. When using the more realistic cross-validation method (data in brackets) the predictive values decrease considerably. Nevertheless the accuracy of the method remains acceptable at threshold levels of $80 \%$ and above. 
Table 4. False predictions ( $50 \%$ level), sharp predictions $(80 \%$ and $90 \%$ threshold levels) and sharp predictions found to be false in the subsequent steps of forward stepwise discriminant analysis

\begin{tabular}{|c|c|c|c|c|c|}
\hline \multirow{2}{*}{$\begin{array}{l}\text { Stepwise } \\
\text { forward } \\
\text { selection }\end{array}$} & \multirow{2}{*}{$\begin{array}{l}50 \% \text { level } \\
\text { predictions }\end{array}$} & \multicolumn{2}{|c|}{$80 \%$ level } & \multicolumn{2}{|c|}{$90 \%$ level } \\
\hline & & $\begin{array}{l}\% \text { of sharp } \\
\text { predictions }\end{array}$ & $\begin{array}{c}\% \text { of false } \\
\text { predictions }\end{array}$ & $\begin{array}{l}\% \text { of sharp } \\
\text { predictions }\end{array}$ & $\begin{array}{l}\% \text { of false } \\
\text { predictions }\end{array}$ \\
\hline lst step & $33 \%$ & $11 \%$ & $0 \%$ & $11 \%$ & $0 \%$ \\
\hline 2nd step & $31 \%$ & $19 \%$ & $4 \%$ & $18 \%$ & $0 \%$ \\
\hline 3rd step & $29 \%$ & $24 \%$ & $3 \%$ & $18 \%$ & $0 \%$ \\
\hline 4 th step & $26 \%$ & $34 \%$ & $10 \%$ & $20 \%$ & $4 \%$ \\
\hline 5 th step & $23 \%$ & $42 \%$ & $10 \%$ & $23 \%$ & $4 \%$ \\
\hline 6th step & $24 \%$ & $44 \%$ & $10 \%$ & $28 \%$ & $3 \%$ \\
\hline 7 th step & $25 \%$ & $48 \%$ & $5 \%$ & $29 \%$ & $0 \%$ \\
\hline
\end{tabular}

Table 5. Predictive values of recurrence and non-recurrence al various threshold levels

\begin{tabular}{|c|c|c|c|c|c|c|c|}
\hline \multirow[b]{2}{*}{$\begin{array}{l}\text { Threshold } \\
\text { level }\end{array}$} & \multicolumn{3}{|c|}{ Prediction of recurrence } & \multicolumn{3}{|c|}{ Prediction of non-recurrence } & \multirow{2}{*}{$\begin{array}{l}\text { Total No. } \\
\text { of patients } \\
\text { assigned }\end{array}$} \\
\hline & Correct & False & $\begin{array}{c}\text { Predictive } \\
\text { value }\end{array}$ & Correct & False & $\begin{array}{l}\text { Predictive } \\
\text { value }\end{array}$ & \\
\hline $50 \%$ & $34(30)^{*}$ & $15(22)$ & $69 \%(58 \%)$ & $55(48)$ & $15(19)$ & $79 \%(72 \%)$ & $119(119)$ \\
\hline $60 \%$ & $31(27)$ & $13(17)$ & $70 \%(61 \%)$ & $46(43)$ & $13(16)$ & $78 \%(73 \%)$ & $103(103)$ \\
\hline $70 \%$ & $30(26)$ & $9(13)$ & $77 \%(66 \%)$ & $40(33)$ & $6(7)$ & $87 \%(82 \%)$ & $85(79)$ \\
\hline $80 \%$ & $26(21)$ & $2(4)$ & $93 \%(84 \%)$ & $28(26)$ & $1(6)$ & $97 \%(81 \%)$ & $57(57)$ \\
\hline $90 \%$ & $17(17)$ & $0(3)$ & $100 \%(85 \%)$ & $17(18)$ & $0(2)$ & $100 \%(90 \%)$ & $34(40)$ \\
\hline $95 \%$ & $14(15)$ & $0(0)$ & $100 \%(100 \%)$ & $12(11)$ & $0(1)$ & $100 \%(92 \%)$ & $26(27)$ \\
\hline
\end{tabular}

*The results when applying the split-ten method are given in parentheses.

\section{E. Practical application of the prognosis rule}

The discriminant analysis formula for calculating the predictions is difficult to use routinely without a computer. However the formula can be closely approximated by an easy-to-use prediction chart, see Table 6 . The originally multiplicative formula is brought into an additive form by a logarithmic transformation. The log-odds score on recurrence calculated from the chart can be translated back into a probability value by using the accompanying transformation table. The scores in the table reflect log likelihood ratios and have been rounded to integers in order to make calculation easier.

\section{DISCUSSION}

Application of stepwise forward selection of prognostic variables resulted in a prognosis rule based on seven variables. This rule yielded probabilistic predictions with a reasonable degree of accuracy at threshold levels of $80 \%$ or more in $57 \%$ of the future recurrences and $41 \%$ of the non-recurrences (see Tables 1 and 5) adding up to $48 \%$ of all patients. The predictions in the other patients resulted in an unacceptable proportion of errors. In this study carcinomo-embryonic antigen appeared to be the most valuable predictive single marker, followed by C3-proactivator. The significance of CEA as a tumor marker in cervical cancer has been reported by others (for review see Ref. [11]). Although rarely, elevations of C3 PA serum levels in cervical cancer have been reported previously [22]. It probably signifies a non-specific acute phase reactant effect, although a specific role of the alternative complement pathway in the defence against cancer cells cannot be excluded.

The stage of the tumor was only the third most important variable, and it did not add to the prognostic information, once CEA was used, due to the considerable correlation between both variables. There was also a strong association of the stage of disease with the age of the patient, but not with the other selected variables.

Due to the relative lack of specificity and sensitivity of the selected variables, the results are far from ideal especially when using the split-sample technique. Moreover, this most realistic validation method is based on the assumption that patient selection, data collection and assay conditions will not change in future patients. Therefore, the reliability of the prediction rule obtained in this study needs to be verified prospectively in a different clinical setting. Wasson et al. [23] define seven methodological standards for prediction rules: definition of outcome, definition of prognostic factors, patients' age and sex stated, study site described, test of misclassification rate, mathematical technique described and effects of clinical use prospectively measured. With the exception of the 
Table 6 . Prediction chart and transformation table derived from the discriminant analysis formula

\begin{tabular}{|c|c|c|c|c|c|}
\hline \multicolumn{4}{|c|}{ Prediction chart: patient. ...... } & \multicolumn{2}{|c|}{ Transformation table } \\
\hline Variable & Value & $\begin{array}{c}\text { A } \\
\text { Score for } \\
\text { non-recurrence }\end{array}$ & $\begin{array}{l}\text { B } \\
\text { Score for } \\
\text { recurrence }\end{array}$ & $\begin{array}{l}\text { Log odds } \\
(\mathrm{B}-\mathrm{A})\end{array}$ & $\begin{array}{c}\text { Probability of } \\
\text { recurrence/non-recurrence } \\
(\%)\end{array}$ \\
\hline CEA & $\leqslant 2$ & 6 & & 0 & 50 \\
\hline \multirow[t]{2}{*}{$(\mathrm{ng} / \mathrm{ml})$} & $\geqslant 14$ & & 30 & 1 & 53 \\
\hline & & & & 2 & 55 \\
\hline $\mathrm{C}_{3} \mathrm{PA}$ & $\leqslant 16$ & 23 & & 3 & 57 \\
\hline \multirow[t]{2}{*}{$(\mathrm{mg} / 100 \mathrm{ml})$} & $\geqslant 29$ & & 10 & 4 & 60 \\
\hline & & & & 5 & 62 \\
\hline \multirow[t]{3}{*}{$\%$ Bandforms } & 0 & 8 & & 6 & 65 \\
\hline & $\geqslant 4$ & & 7 & 7 & 67 \\
\hline & & & & 8 & 69 \\
\hline \multirow[t]{3}{*}{ Age (years) } & $\leqslant 51$ & 5 & & 9 & 71 \\
\hline & $\geqslant 73$ & & 16 & 10 & 73 \\
\hline & & & & 11 & 75 \\
\hline Lympho-vascular & no & 10 & & 12 & 77 \\
\hline \multirow[t]{2}{*}{ invasion } & yes & & 21 & 13 & 79 \\
\hline & & & & 14 & 80 \\
\hline Reticulocytes & $\leqslant 2$ & 14 & & 15 & 82 \\
\hline \multirow{2}{*}{$(\% \circ)$} & $\geqslant 11$ & & 5 & 16 & 83 \\
\hline & & & & 17 & 85 \\
\hline \multirow{3}{*}{$\begin{array}{l}\text { No. of eosinophils } \\
\quad\left(\times 10^{9} / \mathrm{l}\right)\end{array}$} & $\leqslant 2$ & 8 & & 18 & 86 \\
\hline & $\geqslant 5$ & & 9 & 19 & 87 \\
\hline & & & & 20 & 88 \\
\hline \multirow[t]{3}{*}{ Constant factor } & & & 3 & 21 & 89 \\
\hline & & + & + & 22 & 90 \\
\hline & & - & - & 23 & 91 \\
\hline \multirow{5}{*}{$\begin{array}{l}\text { Add relevant } \\
\text { scores }\end{array}$} & & & & 24,25 & 92 \\
\hline & & A: & B: & 26 & 93 \\
\hline & & & & 27,28 & 94 \\
\hline & & & & 29,30 & 95 \\
\hline & & & & $31-33$ & 96 \\
\hline Final score $=\mathrm{B}-\mathrm{A}$ : & & & & $34-36$ & 97 \\
\hline \multirow{3}{*}{ (log odds on recurrence) } & & & & $37-41$ & 98 \\
\hline & & & & $42-50$ & 99 \\
\hline & & & & $>50$ & $99.5-100$ \\
\hline
\end{tabular}

Use: circle the relevant $\mathrm{A}$ and $\mathrm{B}$ scores and add. Final score $=\mathrm{B}-\mathrm{A}=\log$ odds on recurrence. Look in transformation table for translation from $/ B-A /$ (absolute value) to probability. Then: $B-A=$ positive: probability of recurrence (complement: prob. of nonrecurrence). $B-A=$ negative: probability of non-recurrence (complement: prob. of recurrence).

Example: patient has following values: $\mathrm{CEA}<2, \mathrm{C}_{3} \mathrm{PA}=34, \%$ bandforms $=6$, age $=55$, no lymphovascular invasion, reticulocytes $=2$, eosinophils $=4$. Circle associated scores and add. A scores: 6 (for CEA) +10 (for lymphovascular invasion) +14 (for reticulocytes $)=30$. B. scores: 10 (for $\left.\mathrm{C}_{3} \mathrm{PA}\right)+7$ (for $\%$ bandforms $)+3$ (constant factor) $=20$ (both age and ocsinophils do not enter). Final score $=B-A=-10$. Look up ' 10 ' in transformation table for associated probability $=73 \%$. Conclusion: probability of nonrecurrence is $73 \%$ (of recurrence $27 \%$ ).

last one, the present paper satisfies these standards. We have presented the results in such a way that prospective use and evaluation can easily be performed.

At present we can only speculate whether predictive information obtained before treatment is potentially beneficial for patients with cervical carcinoma. This has to be considered separately for non-recurrence and recurrence patients.

Since the available treatment modalities bear considerable risks, one may envisage less radical treatment for the predicted non-recurrences. With regard to the patients treated by radiotherapy, no information is available as to the extent of disease apart from the results of clinical staging. Therefore, the predictive information does not allow any conclusion as to what part of the radiotherapy protocol might be superfluous. For the patients treated by operation one may consider less radical treatment in terms of (partly) omitting lymphadenectomy or compromising on the radicality of the extirpation of the parametria. However, when analyzing the results of the predicted non-recurrence patients at the $95 \%$ threshold level (Table 5), two of the 12 patients appeared to harbor positive pelvic lymph nodes. Both of them were correctly predicted, but they might have developed recurrent disease if less radical treatment had been employed. A trial of less 
radical treatment of the predicted non-recurrence patient seems therefore not justified at present. Apart from the psychological advantage of knowing to be almost certainly cured, the prediction of non-recurrence does not warrant to change the conventional treatment protocol at present. What are the possible advantages for the predicted recurrences? The limits of radicality for local treatment (i.e. treatment solely applied to the pelvis) have probably been reached, both for surgery and radiotherapy $[11,12,24,25]$. A major proportion of failures concern patients who have disseminated disease, whether or not in combination with a loco-regional recurrence [26]. The vast majority of distant metastases are bound to be present (not yet detectable) at the time of primary treatment [11]. Therefore, systemic therapy subsequent to primary treatment might be valuable for the elimination of undetectable metastases. It is feasible that such treatment might also assist in the prevention of locoregional recurrence. Multi-agent chemotherapy in which cis-platinum has been included is probably the best alternative for adjuvant therapy available at present $[27,28]$. The prediction of recurrence might therefore be of advantage for the patient. Whether this is true or not has to be clarified in a prospective randomized study of predicted recurrences where the survival of half of the patients receiving adjuvant chemotherapy is compared to the survival of patients still being treated in the conventional way. $\mathrm{lt}$ is expected that large numbers of patients followed for many years are to be involved in such a trial before it can be concluded whether or not cure rates will improve.

\section{REFERENCES}

1. Kottmeier HL. Carcinoma of the cervix. In: Kottmeier HL, ed. Annual Report on the Results of Treatment in Gynaecologic Cancer. F.I.G.O., 1979, vol. 17, pp. 37-44.

2. Ng AB, Atkin NB. Histological cell type and DNA value in the prognosis of squamous cell cancer of the uterine cervix. Cancer 1973, 28, 322-331.

3. van Nagell JR, Donaldson EG, Wood EG, Parker JG. The significance of vascular invasion and lymfocytic infiltration in invasive cervical cancer. Cancer 1978, 41, 228-234.

4. van Nagell JR, Donaldson EG, Parker JG, van Dijke AH, Wood EG. The prognostic significance of cell type and lesion size in patients with cervical cancer treated by radical surgery. Gynecol Oncol 1977, 5, 142-148.

5. Chung CK, Nahhas WA, Stryker JA. Analysis of factors contributing to treatment failures in stages IB and IIA carcinoma of the cervix. Am J Obstet Gynecol 1980, 138, 550-556.

6. Homesley HD, Raben M, Blake DD. Relationship of lesion size to survival in patients with stage IB squamous cell carcinoma of the cervix uteri treated with radiation therapy. Surg Gynaecol Onstet 1980, 150, 529-531.

7. Kolbenstvedt A. Lymphography in the diagnosis of metastases from carcinoma of the uterine cervix stages I and II. Acta Radiol Diagn 1975, 16, 81-97.

8. Kilcheski ThS, Arger PH, Mulhern ChB, Coleman BG, Kressel HY, Mikuta JI. Role of computed tomography in the presurgical evaluation of carcinoma of the cervix. $J$ Comput Assist Tomogr 1981, 5, 381-383.

9. Ginaldi S, Wallace S, Jing B, Bernardino ME. Carcinoma of the cervix: lymphangiography and computed tomography. Am J Roentgenol 1981, 136, 1087-1091.

10. Rotman M, John M, Boyce J. Prognostic factors in cervical carcinoma: implications in staging and management. Cancer 1981, 48, 560-567.

11. te Velde ER, Persijn JP, Ballieux RE, Faber J. Carcinoembryonic antigen serum levels in patients with squamous cell carcinoma of the uterine cervix: clinical significance. Cancer 1982, 49, 1866-1873.

12. te Velde ER. The clinical significance of tumor index substances and the assessment of general immune competence in patients with cervical cancer. Thesis Univesity of Utrecht, the Netherlands, 1982.

13. Wentr. WB, Reagan JW. Survival in cervical cancer with respect to cell type. Cancer 1959 , 12, 384-388.

14. Lusted LB. Introduction to Medical Decision Making. Springfield, Ch C Thomas, 1968.

15. Titterington DM, Murray GD, Spiegelhalter DJ et al. Comparison of discrimination applied to a complex data set of head injured patients. J R Stat Soc A 1981, 144, 145-175.

16. Habbema JDF, Hermans J. Selection of variables in discriminant analysis by $F$-statistic and error rate. Technometrics $1977, \mathbf{1 9}, 487-493$.

17. Habbema JDF, Gelpke GJ. A computer program for selection of variables in diagnostic and prognostic problems. Comp Prog Biomed 1981, 13, 251-270.

18. Braakman R, Gelpke GJ, Habbema JDF, Mass AIR, Minderhoud JM. Systematic selection of prognostic features in patients with severe head injury. Neurosurgery 1980, 6, 362-370.

19. Hilden J, Habbema JDF, Bjerregaard B. The measurement of performance in probabilistic diagnosis: III methods based on continuous functions of the diagnostic probabilities. Meth Inform Med 1978, 17, 238-246.

20. Habbema JDF, Hilden J. The measurement of performance in probabilistic diagnosis: IV utility considerations in therapeutics and prognostics. Meth Inform Med 1981, 20, 80-96. 
21. Habbema JDF, Hermans J, van de Burgt AT. Cases of doubt in allocation problems. Biometrika 1974, 61, 313-324.

22. te Velde ER, Berrens L, Zegers BJM, Ballieux RE. Acute phase reactants and complement components as indicators of recurrence in human cervical cancer. Eur J Cancer 1979, 15, 893-899.

23. Wasson JH, Sox HC, Neff RK, Goldman L. Clinical prediction rules. N Engl J Med 1985, 313, 793-799.

24. Tewfik HH, Buchsbaum HJ, Latourette HB, Lifshitz SG, Tewfik FA. Para-aortic lymph node irradiation in carcinoma of the cervix after exploratory laparotomy and biopsy-proven positive para-aortic nodes. Int J Radiat Oncol Biol Phys 1982, 8, 13.

25. Potish RA, Twiggs LB, Adcock LL, Prem KA. The utility and limitations of decision theory in the utilization of surgical staging and extended field radiotherapy in cervical cancer. Obstet Gynaecol Survey 1984, 39, 555-562.

26. Paunier JP, Delclos L, Fletcher GH. Causes, time of death, and sites of failure in squamous cell carcinoma of the uterine cervix on intact uterus. Radiology 1967, 88, 555-562.

27. Alberts DS, Martimbeau PW, Surwitz ON. Mitomycin-C, bleomycin, vincristine, cisplatin in the treatment of advanced, recurrent squamous cell carcinoma of the cervix. Cancer Clin Trials 1981, 4, 313-316.

28. Piver MS, Barlow JJ, Lele SB, Maniccia RN. Weckly cis-diamminedichloroplatinum II as induction chemotherapy in recurrent carcinoma of the cervix. Gynaecol Oncol 1984, 18, 313-319. 